PHYSICAL REVIEW D 95, 119903(E) (2017)

\title{
Erratum: Polarization observables for millicharged particles in photon collisions \\ [Phys. Rev. D 94, 095014 (2016)]
}

\author{
Emidio Gabrielli, Luca Marzola, Edoardo Milotti, and Hardi Veermäe \\ (Received 29 May 2017; published 26 June 2017)
}

DOI: $10.1103 /$ PhysRevD.95.119903

Here we clarify, revise and expand the experimental scheme that we proposed in original paper.

In the original scheme, the dark fringe is defined by the condition $\varphi=\pi$ so that the interference is normally destructive, i.e.,

$$
I_{\mathrm{tot}}=I_{1}+I_{2}-2 \sqrt{I_{1} I_{2}},
$$

where the subscripts 1 and 2 denote the two parts of the split beam: 1 corresponds to the part that does not interact with the probe beam, 2 is the part that can be affected by the interaction with the probe beam. These two parts can be further individually modulated (both in amplitude and phase) before interfering on the detector plane. When the beams are static and exactly balanced we have $I_{1}=I_{2}=I_{0}$, so that $I_{\mathrm{tot}}=0$. To avoid confusion, here all of the variables that deal with the probe beam have the subscript "probe" (instead of " 2 " as in our original publication).

The actual beams are off balance because of beam modulation, noise, and, hopefully, real physics. In order to extract the physical signal two different beam modulation schemes are possible. In the first scheme one modulates beam 1 so that

$$
\begin{aligned}
& I_{1}=I_{0}-m I_{0}\left[1-\cos \left(\omega_{1} t+\varphi_{1}\right)\right] \\
& I_{2}=I_{0}-\delta I .
\end{aligned}
$$

Then,

$$
I_{\text {tot }}=2 I_{0}-m I_{0}\left[1-\cos \left(\omega_{1} t+\varphi_{1}\right)\right]-\delta I-2 \sqrt{\left\{I_{0}-m I_{0}\left[1-\cos \left(\omega_{1} t+\varphi_{1}\right)\right]\right\}\left(I_{0}-\delta I\right)} .
$$

When we take a small modulation index $m \ll 1$, the lhs in Eq. (3) can be expanded as follows,

$$
\begin{aligned}
I_{\mathrm{tot}} & \approx 2 I_{0}-m I_{0}\left[1-\cos \left(\omega_{1} t+\varphi_{1}\right)\right]-\delta I-2 I_{0} \sqrt{1-m\left[1-\cos \left(\omega_{1} t+\varphi_{1}\right)\right]-\frac{\delta I}{I_{0}}} \\
& \approx \frac{I_{0}}{4}\left(m\left[1-\cos \left(\omega_{1} t+\varphi_{1}\right)\right]+\frac{\delta I}{I_{0}}\right)^{2} \\
& \approx \frac{m^{2} I_{0}}{4}\left[1-\cos \left(\omega_{1} t+\varphi_{1}\right)\right]^{2}+\frac{m}{2}\left[1-\cos \left(\omega_{1} t+\varphi_{1}\right)\right] \delta I .
\end{aligned}
$$

The second term in expression (4c) is linear in $\delta I$. Then, we see that by modulating the probe beam as well, as in our original proposal, so that $\delta I$ has the same time dependence as the probe beam $\cos \left(\omega_{\text {probe }} t+\varphi_{\text {probe }}\right)$, the power spectrum of the photodiode signal has a main peak at frequency $\omega_{2}$, and it is surrounded by two sidebands at frequencies $\omega_{2} \pm \omega_{\text {probe }}$, with amplitudes

$$
\frac{\sigma \lambda}{4 h c^{2}} f_{1} I_{0} I_{\text {probe }} m m_{\text {probe }} \text {. }
$$

This is the same result found in original paper, except for a factor $m / 4$. Since $m$ can be of order 1 , and taking into account the likely rise in power output of future lasers, the considerations on the signal to noise ratio are essentially unchanged with respect to original paper. 
In the second modulation scheme, one modulates beam 2 after the interaction region, so that

$$
\begin{aligned}
& I_{1}=I_{0} \\
& I_{2}=\left(I_{0}-\delta I\right)-m\left(I_{0}-\delta I\right)\left[1-\cos \left(\omega_{2} t+\varphi_{2}\right)\right]=I_{0}-m\left(I_{0}-\delta I\right)\left[1-\cos \left(\omega_{2} t+\varphi_{2}\right)\right]-\delta I .
\end{aligned}
$$

Then, when we consider the irradiance of Eq. (3), and we assume that $m \ll 1$ so that it is easy to expand it in series and pinpoint the strongest Fourier components, ${ }^{1}$ we find

$$
\begin{aligned}
I_{\mathrm{tot}} & \approx \frac{I_{0}}{4}\left(m \frac{\left(I_{0}-\delta I\right)}{I_{0}}\left[1-\cos \left(\omega_{2} t+\varphi_{2}\right)\right]+\frac{\delta I}{I_{0}}\right)^{2} \\
& \approx \frac{m^{2} I_{0}}{4}\left[1-\cos \left(\omega_{2} t+\varphi_{2}\right)\right]^{2}+\frac{m \delta I}{8}\left[1-\cos \left(\omega_{2} t+\varphi_{2}\right)\right]\left\{4-m\left[1-\cos \left(\omega_{2} t+\varphi_{2}\right)\right]\right\} .
\end{aligned}
$$

In this second modulation scheme, there are again the same sidebands as in the first scheme- - with amplitude reduced by a factor $(1-m)$, and with additional sidebands that drain some of the power from the closest sidebands. This scheme is more complex to implement experimentally, and has a less convenient power spectrum. The conclusion is that it is desirable to modulate the light through path 1 only. The modified experimental layout is shown in the Fig. 1.

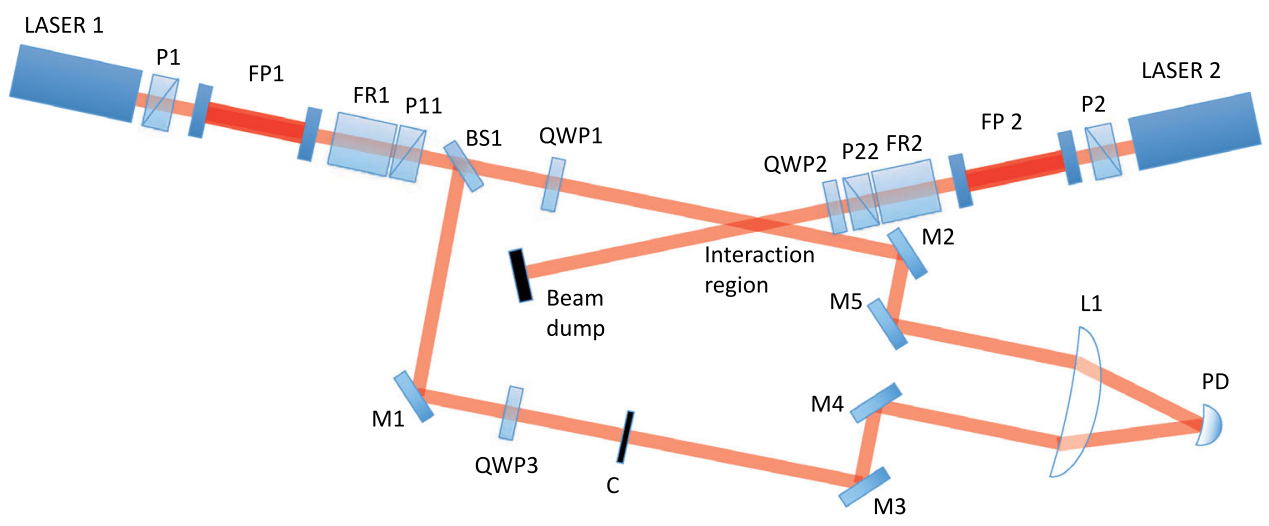

FIG. 1. A possible experimental layout. Two laser beams (the main beam LASER 1 and the probe beam LASER 2) are linearly polarized by the polarizers P1 and P2. The Fabry-Perot cavities, FP1 and FP2, are used to reduce the pointing noise of each laser and as components of a frequency-stabilizing scheme of the Pound-Drever-Hall type. Notice that here frequency stabilization matters because of the interferometric detection scheme. The FR1 and FR2 Faraday cells and the polarizers P11 and P22 are used as amplitude modulators, while the quarter-wave plates, QWP1-QWP3, produce circularly polarized beams. The polarizations are left- or righthanded according to the position of the slow and fast axes of the QWPs. The main beam from LASER 1 is split by a beam splitter, BS1, into two beams that are balanced as much as possible (the optical elements used for balancing are omitted), where M1-M5 are optical mirrors. The chopper, C, introduces a straightforward, additional amplitude modulation on the path of the light that does not interact with the probe beam. The beams are combined again by the convex lens L1 and the optical path lengths are tuned-for instance, by moving mirror M5 relative to M2 - to have a dark fringe on the photodiode PD. Intensity changes in one of the two balanced beams are detected as deviations from the dark fringe condition.

\footnotetext{
${ }^{1}$ We do this for the sake of the expansion; there is no real reason to limit $m$ to extremely low values in the experiment. This means that in general we must consider $m \gg \delta I / I_{0}$, and for this reason we do not drop quadratic terms in $m$, while we keep only terms that are linear in $\delta I$.
} 